\title{
Efficient Message Transfer Using Self Adaptive on Demand Geographic Reliable Routing Algorithm for Mobile AD Hoc Network
}

\author{
Devan.C ${ }^{1}$, Raja.J ${ }^{2}$ \\ PG Scholar, (M.E-Communication \& Networking) Mahendra Engineering College, Namakkal, India ${ }^{1}$ \\ Assistant Professor, CSE, Mahendra Engineering College, Namakkal, India ${ }^{2}$
}

\begin{abstract}
: it has been a big challenge to develop a routing protocol that can meet different application needs and optimize routing paths according to the topology changes in mobile ad hoc networks. Basing their forwarding decisions only on local topology, geographic routing protocols have drawn a lot of attentions in recent years. However, there is a lack of holistic design for geographic routing to be more efficient and robust in a dynamic environment. Inaccurate local and destination position information can lead to inefficient geographic forwarding and even routing failure. The use of proactive fixed-interval beaconing to distribute local positions introduces high overhead when there is no traffic and cannot capture the topology changes under high mobility. It is also difficult to preset protocol parameters correctly to fit in different environments. In this work, we propose two self-adaptive on-demand geographic routing schemes which build efficient paths based on the need of user applications and adapt to various scenarios to provide efficient and reliable routing. To alleviate the impact due to inaccurate local topology knowledge, the topology information is updated at a node in a timely manner according to network dynamics and traffic demand. On-demand routing mechanism in both protocols reduces control overhead compared to the proactive schemes which are normally adopted in current geographic routing protocols. Additionally, our route optimization scheme adapts the routing path according to both topology changes and actual data traffic requirements. Furthermore, adaptive parameter setting scheme is introduced to allow each node to determine and adjust the protocol parameter values independently according to different network environments, data traffic conditions, and node's own conditions. Our simulation studies demonstrate that the proposed routing protocols are more robust and outperform the existing geographic routing protocol and conventional on-demand routing protocols under various conditions including different mobilities, node densities, traffic loads, and destination position inaccuracies. Specifically, the proposed protocols could reduce the packet delivery latency up to 80 percent as compared to GPSR at high mobility. Both routing protocols could achieve about 98 percent delivery ratios, avoid incurring unnecessary control overhead, have very low forwarding overhead and transmission delay in all test scenarios.
\end{abstract}

\section{INTRODUCTION}

There are increasing interests and use of mobile ad hoc networks with the fast progress of computing techniques and wireless networking techniques. In a mobile ad hoc network (MANET), wireless devices could self-configure and form a network with an arbitrary topology. The network's topology may change rapidly and unpredictably. Such a network may operate in a stand-alone fashion, or may be connected to the larger Internet. Mobile ad hoc networks became a popular subject for research in recent years, and various studies have been made to increase the performance of ad hoc networks and support more advanced mobile computing and applications in Mobile ad hoc networks. Even though geographic routing has many advantages and has shown a great potential, the inaccurate knowledge of local geographic topology and destination position can greatly affect routing performance. This not only leads to a larger packet delivery latency and more collisions, but can also result in a routing failure. To obtain the local geographic topology, each mobile node in current geo-graphic routing protocols periodically broadcasts a beacon containing its position. Such a proactive mechanism not only creates a lot of control overhead when there is no traffic, but also results in

"outdated" topology knowledge under high dynamics. To obtain more accurate topology, one option is to determine the beaconing cycle by a moving distance threshold, and another option is to increase the beaconing frequency. For example in with a promiscuous use of the wireless network interface, data packets also serve as beacons. However, with this approach, only the positions of the current forwarding nodes get updated more frequently, but they may no longer be the optimal forwarding nodes as topology changes.

Both options do not consider the actual traffic conditions and routing requirements, and blindly increasing the beaconing frequency may even generate unnecessary overhead. On the other hand, beaconless schemes have been proposed to find the next-hop forwarders in the absence of beacons before each packet transmission. Although this avoids the overhead of sending periodic beacons when there is no traffic, the search of next-hop forwarder before each packet sending introduces a high overhead and end-to-end delay during packet transmissions. In addition to the problems due to beacons, relying on only one-hop topology information in current 
geographic routings may lead to no optimal forwarding and blind forwarding. Furthermore, it is hard to preset the routing parameters to the correct values for all scenarios, which will impact routing performance.

The two protocols adopt different schemes to obtain topology information. One protocol purely relies on onehop topology information as other geographic routing schemes, and the other one assumes a hybrid scheme which combines geographic and topology-based mechanisms for more efficient routing. The use of hybrid scheme avoids the performance degradation of conventional geographic routing by not constraining to local view of topology, and takes advantage of geographic information to find each next-hop thus significantly reducing the overhead and delay incurred by networkrange search of end-to-end path in conventional topologybased on-demand routing.

The position information has the following three sources which all impact routing performance, with the first two assumed to be known and the third one contained in geographic routing protocols: 1) positioning system (e.g., GPS): each node can be aware of its own position through a positioning system, which may have measurement inaccuracy. 2) Location service: every node reports its position periodically to location servers located on one or a set of nodes. The destination positions obtained through these servers are based on node position reports from the previous cycle and may be outdated. 3) Local position distribution mechanism: every node periodically distributes its position to its neighbors so that a node can get knowledge of the local topology. Recently, the impact of the position inaccuracy from the first source has been studied in and the second one is discussed.

Being an important self-contained part of geographic routing protocols, the design of position distribution mechanism will affect local topology knowledge and hence geographic forwarding, but little work has been done to study and avoid its negative impact. Conducts a simulation-based study on the negative effect of mobilityinduced location error on routing performance. Instead, we make a quantitative analysis on the negative effect. Most importantly, we propose two on demand adaptive geographic routing protocols that can meet different application and traffic needs and adapt to different conditions. Our routing schemes are designed to be efficient and robust, with adaptive parameter settings, flexible position distributions, and route optimization.

To summarize, our contributions in this work include:

- Analyzing the effect of outdated topology information on the performance of geographic routing;

- Proposing two novel geographic routing protocols with different schemes to obtain and maintain topology information based on the need of traffic transmissions;

- Introducing route optimization schemes, and to our best knowledge, this is the first geographic routing scheme that adapts the path to the underlying topology change and traffic demand;

- Designing an efficient position distribution mechanism that can adapt its behavior under different dynamics and routing requirements to provide more accurate and updated geographic topology information for efficient routing while reducing unnecessary control overhead; Adapting parameter settings in both protocols ac-cording to different criteria, such as network environment, traffic demand, and node's own condition;

- Handling the inaccuracy of destination position and efficiently avoiding delivery failure.

The rest of this paper is organized as follows: In Section 2, we discuss some related work. Section 3 makes an analysis on the effect of outdated topology knowledge on geographic routing. We provide detailed descriptions of the two protocols in Section 4, and extensive simulation results and performance studies in Section 5. Finally, Section 6 concludes the paper.

\section{RELATED WORK}

As far as we know, there are no geographic routing protocols that are adaptive to the demand of traffic transmissions. We will discuss literature work related to geographic routing protocols and on-demand routing protocols for MANET. The conventional on demand routing protocols often involve flooding in route discovery phase, which limits their scalability. LAR and DREAM make use of the nodes' position information to reduce the flooding range. In LAR, the flooding of route searching messages is restricted to a request zone which covers the expected zone of the destination. In DREAM, intermediate nodes forward packets to all the neighbors in the direction of the estimated region within which the destination may be located.

Although existing beaconless schemes reduce the overhead due to active beacons, the search of the next-hop forwarder for each packet makes the end-to-end delay of these beaconless schemes significantly higher than that of GPSR. In contrast, our first protocol only needs to search for the next-hop forwarder when the traffic is initiated or when the cached next-hop forwarder cannot be reached. Our estimation scheme and adaptation scheme work together to timely update the next-hop forwarders for optimal routing. Instead of completely removing beacons, in our second protocol, the beacons are sent based on traffic demand and the beacon periods are adapted based on network topology and relative moving speed between a node and its neighbors.

Different from existing beaconless geographic routing schemes which simply consider the forwarding procedures without using beacons, the aim of this paper is to design adaptive and robust packet delivery strategies to suit different network settings and traffic conditions, and ensure routing efficiency as network topology or traffic changes. We also consider parameter adaptation to 
improve trans-mission robustness while minimizing the overhead and ensure reliable transmission when the knowledge on the destination position is inaccurate. Our performance studies demonstrate that our algorithms and protocols achieve higher delivery ratio, lower control overhead and delay, and lower redundant transmissions in all scenarios tested, with the variations of mobility, traffic, node density, and inaccuracy of destination position. The procedures for finding the next-hop forwarders proposed by existing beaconless schemes may be used with our algorithms and protocols, which will help to support more robust and efficient transmissions in various dynamic conditions.

\section{Nonoptimal Routing}

To explain why the outdated local topology knowledge may lead to nonoptimal routing, let us look at the example in Fig. 1a. Node B just moved into A's transmission range, which is unknown to A before B sends out its next beacon message. Without knowing any neighbors closer to the destination $\mathrm{G}, \mathrm{A}$ forwards the packet to node $\mathrm{C}$ then $\mathrm{D}$ by using perimeter forwarding. The greedy forwarding is resumed from $D$ to $E$ until reaching $G$. The resulted path has five hops, while the optimal path between $A$ and $G$ should have only two hops after B bridges the void between A and G. Due to the lack of timely and largerrange topology information, the inaccuracy of the local topology knowledge greatly affects the geographic routing performance.

\section{Forwarding Failures}

In the literature work, a neighbor's information will be removed if not updated within the time-out interval, which is often set to be multiple beacon intervals. As a result, a node may hold outdated neighbor information, thus resulting in forwarding failure. This would lead to packet dropping or rerouting. More severely, before detecting the unreachability, the continuous retransmissions at MAC layer reduce the link throughput and fairness, and increase the collisions. This will further increase the delay and energy consumption.

\section{SELF-ADAPTIVE ON-DEMAND GEOGRAPHIC ROUTING PROTOCOLS}

In this section, we present two Self-adaptive On-demand Geographic Routing (SOGR) schemes. In both schemes, we assume every mobile node is aware of its own position (e.g., through GPS or some in-door localization technique), and a source can obtain the destination's position through some kind of location service.

We also make use of the broadcast feature of wireless network to improve routing performance and assume mobile nodes enable the promiscuous mode on their network interfaces. Introduce their different reactive topology finding and maintenance schemes, the associated next-hop selection and recovery strategies, as well as their parameter adaptation schemes.

Both protocols contain an adaptive route optimization component as presented in, in which the position of a nexthop node is estimated before the transmission to avoid position outdate and transmission failure, and the route is optimized according not only to the topology change but also to the actual data traffic requirements. Additionally, we consider the impact of destination position inaccuracy and discuss the schemes to minimize the delivery failure. For the convenience of presentation, in the remainder of the paper, except when explicitly indicated, F represents the current forwarding node, $\mathrm{D}$ is the destination, $\mathrm{N}$ denotes one of F's neighbors.

\section{Scheme 1: SOGR with Hybrid Reactive Mechanism (SOGR-HR)}

Without proactive beaconing to distribute local topology, a scheme needs to be designed for a forwarding node to find the path to the destination. In SOGR-HR, the next-hop of a forwarding node is determined reactively with the combination of geographic-based and topology based mechanisms. By incorporating topology-based path searching, an important benefit of the proposed scheme is to obtain the topology information at a larger range when necessary to build more efficient routing path, while general geographic routing protocols are usually constrained by their local topology view. Furthermore, the planar graph based geo-graphic routing strategy becomes unpractical under the real physical channel conditions. The use of topology-based routing recovery scheme in SOGR helps overcome such shortcomings of geographic routing.

\section{Geography-Based Greedy Forwarding}

Normally a forwarding node $\mathrm{F}$ will attempt to forward a packet greedily to a neighbor closest to the destination D and closer to $\mathrm{D}$ than itself. When there is no next-hop information cached, $\mathrm{F}$ buffers the packet first and broadcasts a request message.

\section{Scheme 2: SOGR with Geographic-Based Reactive} Mechanism (SOGR-GR)

SOGR-GR depends only on one-hop neighbors' positions to make greedy and perimeter forwarding like other geographic routing protocols. However, it adopts a reactive beaconing mechanism which is adaptive to the traffic need.

The periodic beaconing is triggered only when a node overhears data traffic from its neighbors the first time. The beaconing is stopped if no traffic is heard for a predefined period.

A forwarding node may broadcast a request (REQ) message to trigger its neighbors' beaconing when necessary and the neighbors will have random back off before broadcasting a beacon to avoid collision.

With the neighbor topology information, SOGR-GR takes the same local void recovery method as existing geometric routing protocols to avoid the need of extra searching as in SOGR-HR. In addition, similar to SOGR-HR, the important protocol parameters of SOGR-GR are also set adaptively for optimal performance. 


\section{PERFORMANCE EVALUATION}

In this section, we evaluate the performance of SOGR-HR and SOGR-GR with various moving speeds, node densities, traffic loads, and destination position inaccuracies.

\section{Simulation Overview}

Implemented SOGR-HR and SOGR-GR within the Global Mobile Simulation (GloMoSim) library. Although various schemes have been proposed to address different issues, very few literature studies provide complete protocol design that can be followed for implementation. As our protocols are on-demand and geography-based, for performance evaluations, we compare our protocols with the classic topology-based on-demand routing protocol AODV, LAR, an on-demand routing protocol utilizing position information to restrict the flooding range of route searching, and the geographic routing protocol GPSR. Besides demonstrating the efficiency and robustness of our protocols in dynamic scenarios, further confirm the benefit of using geographic routing.

Study the following metrics:

1. Packet delivery ratio. The ratio of the packets delivered to those originated by CBR sources.

2. Control overhead. The total number of control message transmissions (the forwarding of a control message at each hop is counted as one control transmission) divided by the total number of data packets received.

3. Average number of data packet forwarding per delivered packet. The total number of data packet forwarding accumulated from each hop (including rerouting and retransmissions due to collisions) over the total number of data packets received. Both the nonoptimal routing and rerouting due to unreachable next hop will increase the forwarding overhead.

4. Average end to end delay. The average time interval for the data packets to traverse from the CBR sources to the destinations.

5. Packet delivery ratio. The ratio of the packets delivered to those originated by CBR sources.

To demonstrate the effectiveness of our algorithms and protocols in supporting robust communications under various conditions, we have performed extensive simulations with the variations of mobility and thus the rate of network topology changes, node density, traffic load, and the accuracy level of the destination position.

In each performance study, only the parameter to evaluate is varied, and the remaining parameters are set to the default values.

\section{Simulation Results}

Effect of Moving Speed

Study the impact of mobility on the performance of various protocols by varying the maximum moving speed from 0 to $50 \mathrm{~m} / \mathrm{s}$.
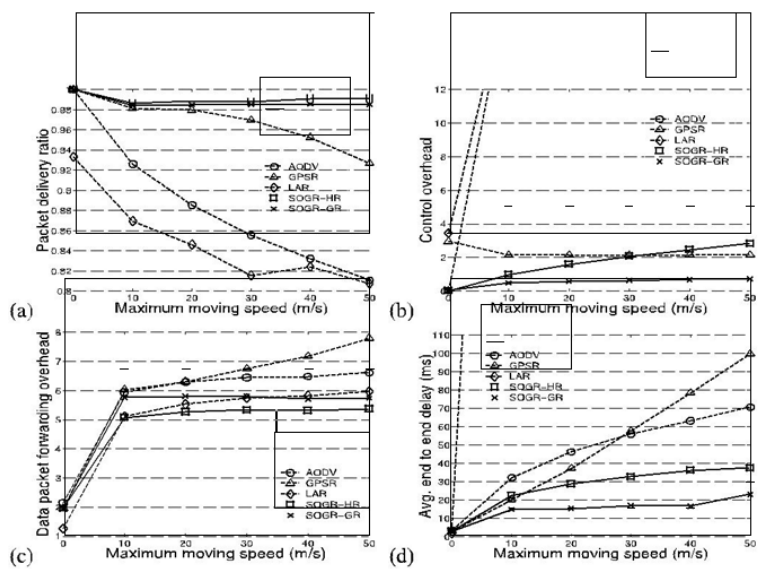

Fig. 1. Performance with different maximum moving speeds (300 nodes, $3 ; 000 \mathrm{~m}_{-}$1;500 m, 30CBR): (a) packet delivery ratio; (b) control overhead;

(c) average number of data packet forwarding; (d) average end to end delay.

from 0 to $50 \mathrm{~m} / \mathrm{s}$. In Fig. 1a, the delivery ratios of the two topology-based protocols drop quickly as the moving speed increases. As mentioned previously, the scalability of LAR and AODV is limited by the involved networkrange or restricted range flooding. The end-to-end paths obtained during route discovery phases are easily broken under network dynamics resulting in packet droppings, although a smaller-range recovery may be initiated after routing failure at the cost of retransmissions and extra control overhead. In contrast, the geographic routing protocols determine the next hop based only on the knowledge of local topology, and can hence respond to the mobility faster. Therefore, all three geographic routing protocols have much higher delivery ratios. SOGR-HR and SOGR-GR maintain a high delivery ratio around 99 percent even in a highly dynamic environment, while the delivery ratio of GPSR drops quickly when the maximum moving speed is higher than $20 \mathrm{~m} / \mathrm{s}$. The stable performance of SOGR-HR and SOGR-GR demonstrates the effectiveness of their adaptive schemes in response to changes of network topology as a result of mobility. When mobile nodes move faster, the local topology information advertised through fixed-interval beaconing in GPSR is more vulnerable to be invalid. While in SOGR-HR and SOGR-GR, the adaptive parameter settings and more flexible position distributions will intelligently generate necessary control messages to dis-tribute position information and better track mobility.

The use of adaptive position update in the two SOGR protocols is verified by Fig. 1b, where both SOGR-HR and SOGR-GR intelligently generate more control messages to capture the topology changes as mobility increases. With a fixed beaconing interval, GPSR has unnecessary control overhead when the mobility is low, and suffers from outdated topology knowledge when the mobility is high. SOGR-HR is seen to generate a slightly higher control overhead than SOGR-GR. In SOGR-HR, whenever the next hop is invalid, the forwarding node will start a new 
route search phase; while in SOGR-GR, the forwarding node just needs to select another valid next hop from its neighbor table without incurring extra control overhead. The increase of mobility leads to a higher chance of path breakage and thus a higher number of path search messages. As a result, the control overheads of AODV and LAR are significantly higher with the increase of mobility.

As expected, GPSR needs more packet forwarding to deliver a packet as shown in Fig. 1c, due to its nonoptimal routing and rerouting caused by the outdated local topology knowledge and the longer routing path during perimeter forwarding. The number of forwarding increases almost linearly with the increase of moving speed. As AODV and LAR usually search for the shortest path to the destination, they have fewer forwarding. SOGR-HR has the fewest forwarding in most cases, and both SOGR-HR and SOGR-GR have much fewer forwarding under high mobility as compared to GPSR. These are due to their use of more efficient position distribution mechanisms to reduce the rerouting of undeliverable packets and route optimization schemes to adapt the route more quickly to the topology changes. SOGR-GR has a little more forwarding than SOGR-HR because as GPSR, the perimeter forwarding in SOGR-GR may introduce more packet forwarding, while by considering topology at a larger range during recovery forwarding, SOGR-HR can build more efficient routing path without being constrained to one-hop information.

In Fig. 1d, LAR and AODV are seen to have a longer endto-end delay due to more frequent path breakage and the time required to rebuild the path before packet forwarding in traditional on-demand routing protocols. The end-to-end delay of GPSR increases almost linearly as mobility increases

Therefore, SOGR-HR and SOGR-GR are robust to the inaccuracy of destination positions and support more reliable packet delivery, while GPSR has a quick decrease of delivery ratio, and increase of packet forwarding overhead and delay as the inaccuracy increases.

\section{CONCLUSIONS}

Developed efficient and robust geographic routing schemes that can be applied for applications with different traffic patterns and adapt to various scenarios to provide efficient routing paths and improve routing performance in a dynamic resource-constrained wireless ad hoc network. Specifically, Occur to two self-adaptive on-demand geographic routing protocols SOGR-HR and SOGR-GR. The two protocols adopt different schemes to obtain and maintain local topology information. SOGR-GR purely relies on one-hop topology information for forwarding as other geographic routing schemes; SOGR-HR combines both geographic and topology based mechanisms for more efficient path building.

The next-hop relay node selection can base on both geographic information and conditions of transmission channels. Investigate the performance of our protocols by incorporating more factors are solved in this work.

The two protocols are designed with the following features:

1. Both protocols incorporate routing parameter adaptations, where each node can determine and adjust its protocol parameter values independently according to mobility, node distributions, and data traffic conditions;

2. To avoid unnecessary control overhead, both protocols distribute topology information and search for routing path only when there is traffic;

3. To alleviate the negative effects of outdated local topology information on geographic routing, more efficient position distribution mechanisms are included to update the local topology in time and adaptively based on traffic demand, and position estimation is used to remove outdated topology records;

4. Optimization schemes are applied so that a forwarding node and its neighbors can collaborate to adapt the path to both topology change and traffic demand and thus improve transmission path opportunistically;

5. Both proposed routing schemes could better deal with the inaccuracy of destination position and its resulting routing inefficiency and failure.

The simulation results demonstrate that our protocols are very robust in a dynamic mobile ad hoc network, and can efficiently adapt to different scenarios and perform better than existing geographic routing protocols and conventional on-demand protocols under various environments, including different mobility, node densities, traffic loads, and destination position inaccuracies. Specifically, compared to GPSR, both SOGR protocols are much more robust to quick topology changes and the inaccuracy of destination positions, and could reduce the end-to-end delay up to 80 percent in high-mobility scenario. Both proposed routing protocols could achieve about 98 percent delivery ratios; avoid incurring unnecessary control over-head, very low-forwarding overhead and transmission delay in all test scenarios. Additionally, SOGR-HR makes a better balance between control overhead and routing path efficiency in a sparse network and in a light-load scenario, and could reduce the packet forwarding overhead up to 86 and 41 percent, respectively, without incurring unnecessary control overhead.

\section{REFERENCES}

[1] [1] B. Karp, "Greedy Perimeter Stateless Routing (GPSR)," http:/www.icir.org/bkarp/gpsr/gpsr.html, 2012

[2] [25].Efficient Beaconless Geo-routing with Guaranteed Delivery in Wireless Sensor, Ad Hoc, and Actuator Networks," IEEE/ACM Trans. Networking, vol. 18, no. 1, pp. 95-108, Feb. 2010.

[3] [3] H. Zhang and H. Shen, "Energy-Efficient Beaconless Geographic Routing in Wireless Sensor Networks," IEEE Trans. Parallel and Distributed Systems, vol. 21, no. 6, pp. 881-896, June 2010

[4] W. Xi, Y. He, Y. Liu, J. Zhao, L. Mo, Z. Yang, J. Wang, and X.-Y. 
Li, "Locating Sensors in the Wild: Pursuit of Ranging Quality," Proc. ACM Conf. Embedded Networked Sensor Systems, 2010.

[5] . Yang, Y. Liu, and X.-Y. Li, "Beyond Trilateration: On the Localizability of Wireless Ad-Hoc Networks," IEEE/ACM Trans. Networking, vol. 18, no. 6, pp. 1806-1814, Dec. 2010.

[6] Y. Li, Y. Yang, and X. Lu, "Routing Metric Designs for Greedy, Face and Combined-Greedy-Face Routing," Proc. IEEE INFOCOM, pp. 64-72, Apr. 2009.

[7] F. Kuhn, R. Wattenhofer, and A. Zollinger, "An Algorithmic Approach to Geographic Routing in Ad Hoc and Sensor Networks," IEEE/ACM Trans. Networking, vol. 16, no. 1, pp. 51-62, Feb. 2008.

[8] X. Xiang and X. Wang, "A Scalable Geographic Service Provision Framework for Mobile Ad Hoc Networks" Proc. IEEE Int'l Conf. Pervasive Computing and Comm., Mar. 2007

[9] X. Xiang, X. Wang, and Z. Zhou, "Robust and Scalable Geographic Multicast Protocol for Mobile Ad Hoc Networks," Proc. IEEE INFOCOM, May 2007

[10] J.A. Sanchez, R. Marin-Perez, and P.M. Ruiz, "BOSS: Beacon-Less on Demand Strategy for Geographic Routing In wireless Sensor Networks," Proc. Fourth Int'l Conf. Mobile Ad-Hoc and Sensor Systems, p. 110, 2007. 